\title{
Dislocation-based Serrated Plastic Flow of High Entropy Alloys at Cryogenic Temperatures
}

\author{
A. S. Tirunilai ${ }^{\mathrm{a}}$, T. Hanemann ${ }^{\mathrm{b}}$, K.-P. Weiss ${ }^{\mathrm{b}}$, J. Freudenberger, ${ }^{\mathrm{c}, \mathrm{d}}$, M. Heilmaier ${ }^{\mathrm{a}}$, A. Kauffmann ${ }^{\mathrm{a}}$ \\ ${ }^{a}$ Institute for Applied Materials (IAM-WK), Karlsruhe Institute of Technology (KIT), Engelbert- \\ Arnold-Str. 4, D-76131 Karlsruhe, Germany \\ ${ }^{b}$ Institute for Technical Physics (ITEP), Karlsruhe Institute of Technology (KIT), Hermann-von- \\ Helmholtz-Platz 1, D-76344 Eggenstein-Leopoldshafen, Germany \\ ${ }^{\mathrm{c}}$ Leibniz Institute for Solid State and Materials Research Dresden (IFW Dresden), Helmholtzstr. 20, D- \\ 01069 Dresden, Germany \\ ${ }^{\mathrm{d}}$ Institute of Materials Science, Technische Universität Bergakademie Freiberg, Gustav-Zeuner-Str. 5, \\ 09599 Freiberg, Germany
}

\section{Abstract}

Serrated plastic deformation at temperatures close to $0 \mathrm{~K}$ has been previously reported in some metals and alloys, and is associated with two possible origins: (i) thermomechanical instability or (ii) mechanical instability. While some recent results indicate that serrations are a mechanical dislocation-based phenomenon, a comprehensive model does not exist. CoCrFeMnNi, an expectedly ideal candidate, exhibits severe serrated plastic deformation with large stress drops in excess of $100 \mathrm{MPa}$. Furthermore, it also shows cryogenic serrated plastic deformation at a higher temperature $(35 \mathrm{~K})$ than previously reported for any other alloy. The exacerbated nature of serrated plastic deformation in CoCrFeMnNi led to the following inferences: (i) temperature and dislocation density are indisputable controlling parameters for cryogenic serrated plastic deformation and they cannot supersede each another; (ii) a phenomenological model is elucidated based on the increasing difficulty for cross-slip with decreasing temperature, leading to sudden massive dislocation proliferation event; (iii) the model establishes a gradual transition from completely non-serrated to completely serrated deformation, mediated by cross-slip, as opposed to the conventional model which proposed a discrete transition; (iv) solute dislocation interaction and associated Stacking Fault Energy (SFE) during deformation plays a key role in controlling dislocation constriction and cross-slip and correspondingly serrated plastic deformation; (v) the need/direct influence of deformation twinning, transformation induced plasticity and especially thermomechanical factors on serrated plastic deformation is invalidated. Some of these points were further clarified through comparisons with $\mathrm{CoCrNi}$ and $\mathrm{CoNi}$, also presented in the present article. 


\section{Keywords}

alloy; deformation; high-entropy alloys; serrations; cryogenic temperatures; Lomer-Cottrell lock; crossslip

\section{Introduction}

High Entropy Alloys (HEA) are a category of alloys comprising multiple principle elements that form concentrated solid solutions $[1,2,3]$. These alloys have been a point of intense focus over the course of the last decade $[4,5,6,7,8]$. Among various HEAs, CoCrFeMnNi, also known as the Cantor alloy [9], has served as a model face centered cubic (FCC) alloy $[10,11,12,13,14]$. This alloy is known to exhibit interesting mechanical behavior at cryogenic temperatures, including strong work-hardening with decreasing temperature [15], activation of deformation twinning at cryogenic temperatures [10] and intense serrated plastic deformation at very low temperatures $[16,17]$. The current article focusses on the last of these issues.

Serrated plastic deformation at cryogenic temperatures close to $0 \mathrm{~K}(4-35 \mathrm{~K})$ has been reported for different metals and alloys [18, 19, 20, 21, 22]. An example for this is given in Fig. 1. The phenomenon has been attributed to one of two possible effects: (i) a thermomechanical effect or (ii) a mechanical effect. The first one is based on localized thermal softening. Essentially, at cryogenic temperatures, macroscopic deformation of a specimen is not continuous and takes place through a series of local deformation steps. The local deformation releases heat. The amount of heat released is manifested locally as a temperature spike. The temperature spike is significant due the very low heat capacity $\left(c_{p}\right)$ and thermal conductivity $(\lambda)$ seen in metallic materials close to $0 \mathrm{~K}$ [23]. Low $c_{p}$ makes the temperature spike high and low $\lambda$ keeps the spike local, thus causing local thermal softening. This is manifested as a serration or a drop in the stress. Alternatively, the second mentioned effect is that dislocations pile up at barriers which break down at critical stresses resulting in avalanche slip.

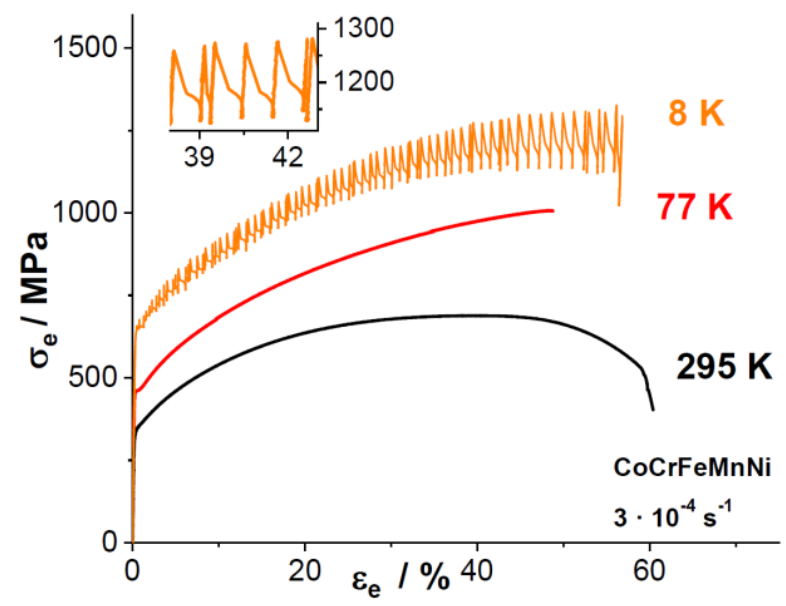

Figure 1: Engineering stress-strain $\left(\sigma_{e}-\varepsilon_{e}\right)$ diagram showing results of tensile tests conducted on $\mathrm{CoCrFeMnNi}$ at $295 \mathrm{~K}, 77 \mathrm{~K}$ and $8 \mathrm{~K}$. The initial engineering strain rate was $\dot{\varepsilon_{e}} \sim 3 \cdot 10^{-4} \mathrm{~s}^{-1}$. A 
detailed discussion of deformation behavior and microstructural evolution can be found in Refs. [15, $16]$.

Avalanche phenomenon, as reported in literature, refer to power-law breakdown events [24]. The model is not only applicable for crystal plasticity but also to natural events such as earthquakes [25]. However, avalanche slip as referred to in the context of cryogenic serrated plastic flow refers to the sudden proliferation and associated movement of a large number of dislocations. In the current context this is associated with the activation of dislocation sources as a result of stress fields from dislocation pile-ups at barriers. Considering that avalanche events are associated with power-law phenomena, this event will instead be referred to as a dislocation proliferation event throughout the article. It is used in the same context as 'avalanche slip' in previous publications on the topic. The first version of the mechanical model was proposed by Seeger [26]. According to this model, screw dislocations leave trails of interstitials or (mainly) vacancies behind after having intersected forest dislocations in contrast to intersecting edge dislocations [27]. This defect formation has an energy associated with it and is predominantly compensated at higher temperatures by thermal activation. Consequently, at lower temperature the amount of external (mechanical) energy for interstitial defect formation becomes increasingly larger. Below a certain temperature, the energy needed for screw dislocation motion is too high. In this temperature range, deformation takes place mainly by edge dislocation motion. This model was further verified by Obst and Nyilas [21] where they showed lack of $\alpha$ '-martensite formation in $316 \mathrm{LN}$ steel below a certain temperature. Martensite formation is consistently associated with screw dislocation motion and, hence, a lack of martensite formation indicates a lack of screw dislocation activity. Dislocations pile up at LC locks, formed during deformation. At very low temperatures, as explained, mobile dislocations are of edge type. These cannot cross-slip out of the pile-up at the lock. At some critical stress at the head of the dislocation pile-up, a dislocation source is activated on the other side of the lock. This can lead to the further activation of multiple sources and the slip associated with it causes the macroscopically observed stress drops.

In order to effectively evaluate serrated plastic deformation one would have to conduct experiments on an alloy that shows the phenomenon in a pronounced fashion. Greater solid solution strengthening correlates positively to this effect [22]. Additionally based on the mechanical model, a metal or alloy with low to medium stacking fault energy would maximize this effect [26]. The probe material should exist as stable single-phase FCC at temperatures as low as $4 \mathrm{~K}$. Two-phase materials cause significant complications in appropriately correlating the results to microstructural phenomenon. The activation of deformation twinning at some stage of deformation is a beneficial, but not necessary, condition. This would help clarify the influence of deformation twinning in the discontinuous nature of deformation, considering that twinning is responsible for stress drops in single crystals [19] and is influential in high temperature discontinuous deformation [28, 29]. 


\section{Experimental}

\section{Synthesis of the material and materials characterization} appropriate, as a probe material to investigate serrated plastic deformation. thermomechanical effect and, thus, are more likely due to a mechanical effect. backdrop whenever necessary.

$\mathrm{CoCrFeMnNi} \mathrm{emphatically} \mathrm{fulfills} \mathrm{all} \mathrm{of} \mathrm{these} \mathrm{requirements.} \mathrm{Austenitic} \mathrm{stainless} \mathrm{steels} \mathrm{also} \mathrm{prove} \mathrm{to} \mathrm{be}$ strong candidates for the study of serrations and have formerly been used to state/qualify multiple hypotheses related to cryogenic serrated plastic deformation [21, 22, 30, 31]. However, (i) austenitic stainless steels show $\alpha$-martensite transformation at cryogenic temperatures, intervening with existing dislocation phenomenon and correspondingly making analysis of dislocation-serration correlation more complex; (ii) some of the austenitic steels also have a non-negligible interstitial content which makes it difficult to both control the composition from batch to batch as well as ensure a homogenous interstitial distribution. CoCrFeMnNi circumnavigates, both issues as a substitutional solid solution with no apparent martensite transformation at cryogenic temperatures [16]. Thus, $\mathrm{CoCrFeMnNi}$ is uniquely

In a previous publication [16], it was established that $\mathrm{CoCrFeMnNi}$ is fairly insensitive to extrinsic experimental conditions. Change in cooling medium and surface area to volume ratio of the gauge section did not correlate with any noticeable change in the stress drop amplitude $\Delta \sigma_{e}$ (the engineering stress change from maxima to following minima of serrations). Additionally, $\Delta \sigma_{e}$ did not change with strain rate below a specific upper limit. All these results correlate with a lack of influence from a

In the current article, the results on the alloys $\mathrm{CoCrFeMnNi}$, $\mathrm{CoCrNi}$ and $\mathrm{CoNi}$ are utilized [15], to establish a phenomenological model on the mechanism behind serrated plastic deformation as well as the temperature based changes resulting in a transition from continuous deformation at higher temperatures to discontinuous deformation at lower temperature. The examination is made through quantitative statistical analysis, considering stress drop amplitudes, time lapses during stress drops and rate of change of stress drops with progression in deformation. Moreover, the possible effects of both, deformation twinning and $\varepsilon$-martensite formation on the serrations are explored. As can be gleaned from the results below, the effect is severe in both $\mathrm{CoCrFeMnNi}$ and $\mathrm{CoCrNi}$. This is a major reason for being able to elucidate certain features in serration behavior that have formerly gone unreported. Additionally, multiple fundamentals of the existing mechanical model have been corrected, while also invalidating some often cited hypotheses. Finally, building on previous report [16] supporting the mechanical model, several results in the current article also invalidate localized thermal softening as the cause for serrations. While special focus is not given to evaluation under this model, the results are analyzed under its

The investigated $\mathrm{CoCrFeMnNi}, \mathrm{CoCrNi}$ and $\mathrm{CoNi}$ samples were synthesized from elemental bulk metals through arc melting. Subsequently, the as-cast samples were homogenized and cold swaged. Tensile specimens with cylindrical cross sections were machined from these cold worked specimens 


\section{Results and Discussion}

\section{Influence of temperature on serrations}

132 The effect of temperature is illustrated through a series of tensile tests conducted on CoCrFeMnNi at $13335 \mathrm{~K}, 25 \mathrm{~K}, 15 \mathrm{~K}, 8 \mathrm{~K}$ and $4.2 \mathrm{~K}$. The corresponding engineering stress-strain $\left(\sigma_{e}-\varepsilon_{e}\right)$ curves are 134

and recrystallized subsequently. A more detailed overview of the procedure can be found in a previous publication [15].

All three alloys were confirmed to be single-phase FCC using X-ray diffraction (XRD). XRD was carried out on recrystallized and polished longitudinal sections of the alloys using a D2 Phaser system by Bruker, equipped with a LynxEye line detector. A fully recrystallized condition was confirmed using Scanning Electron Microscopy (SEM) imaging. SEM using backscatter electron imaging (BSE) and EDS was performed with two different devices, namely a Zeiss Leo 1530 and a Zeiss Auriga 60. A more detailed overview of specimen preparation and the corresponding characterization results has been published in Ref. [15].

\section{Mechanical Testing}

Tensile tests have been performed at room temperature $(295 \mathrm{~K})$ and at different cryogenic temperatures ranging from 35 to $4.2 \mathrm{~K}$. These tests were performed at the Cryogenic Material Test Lab Karlsruhe (CryoMaK) of the Institute of Technical Physics (ITEP, KIT), the process for which is described elsewhere [32]. The cylindrical tensile specimens have M6 connecting threads, a total length of $45 \mathrm{~mm}$, a uniform (gauge) length of $22 \mathrm{~mm}$, a transition radius of $10 \mathrm{~mm}$ and a gauge diameter of $4 \mathrm{~mm}$.

Tensile tests performed down to $8 \mathrm{~K}$ were carried out utilizing an MTS 25 testing device, filled with $\mathrm{He}$ vapor at a pressure $\sim 50$ mbar while the test at $4.2 \mathrm{~K}$ was carried out in a liquid Helium bath using another machine called the ATLAS. Tensile testing was performed until fracture with constant crosshead movement corresponding to an initial plastic strain rate of $\sim 3 \cdot 10^{-4} \mathrm{~s}^{-1}$ under standard conditions according to ASTM E8M. The strain was measured using strain gauges attached to the samples. The acquisition rate used was $100 \mathrm{~Hz}$. Based on the measured force, elongation data and the sample dimensions, other parameters such as stress, strain, work-hardening rate, true stress and true strain values were derived, using the proprietary software package Origin 2018 by OriginLab. Additionally, with reference to the observed serrations, the maxima and minima for each of these serrations were identified using a MATLAB R2018a (MathWorks) script written by the authors which was then used in the estimation of the work-hardening rate. The local stress minimum was estimated after an appropriate adjustment to accommodate machine contributions to the stress drop. A more detailed explanation of this can be found in the Appendix. plotted in Fig. 2a. 



Figure 2: (a) $\sigma_{\mathrm{e}}-\varepsilon_{\mathrm{e}}$ curves of $\mathrm{CoCrFeMnNi}$ deformed at $35 \mathrm{~K}, 25 \mathrm{~K}, 15 \mathrm{~K}, 8 \mathrm{~K}$ and $4.2 \mathrm{~K}$, (b) corresponding $\Delta \sigma_{\mathrm{e}}-\sigma_{\mathrm{e}}$ curves. In (a), the $\sigma_{\mathrm{e}}-\varepsilon_{\mathrm{e}}$ curves are vertically offset to ensure that the reader can resolve the individual features as the curves would otherwise overlap significantly.

The specimen at $25 \mathrm{~K}$ did not rupture during the test as the cross head displacement of the machine had reached its limit. However, considering the significant strain achieved and since this was not a critical factor in the current study the specimen was included in the analysis. Serrations were visible in $\mathrm{CoCrFeMnNi}$ up to the temperature of $35 \mathrm{~K}$. This is the highest temperature of low temperature serrated plastic flow reported thus far. $316 \mathrm{LN}$ steel shows serrations at $34 \mathrm{~K}$ [21], however, the serrations only appear very close to failure and are far too few (3 serrations) to accurately deduce a trend or have representative characteristics. $\mathrm{CoCrFeMnNi}$ on the other hand, shows serrations at $35 \mathrm{~K}$ that are more clearly resolved, from which a trend can be obtained. Additionally, $316 \mathrm{LN}$ shows serrations at $\varepsilon_{e}>$ $5 \%$ at $15 \mathrm{~K}$. At the same temperature, $\mathrm{CoCrFeMnNi}$ shows serrations starting at yield point. The highest temperatures where serrations appear during deformation starting at yield point are reportedly higher for CoCrFeMnNi (15 K) than for $316 \mathrm{LN}$ steel (7 K) [21], which was formerly reported as having one of the highest temperatures of any metallic materials, for the same. This comparison is also noteworthy considering the substantially higher yield strength $\left(\sigma_{\mathrm{YS}}\right)$ of $316 \mathrm{LN}, \sim 1100 \mathrm{MPa}$ at $34 \mathrm{~K}$ [21] in contrast to only $605 \mathrm{MPa}$ for $\mathrm{CoCrFeMnNi}$ at $35 \mathrm{~K}$ [15], implying a higher strength does not automatically imply a higher tendency towards serrated flow.

Fig. 2b shows the estimated $\Delta \sigma_{e}$ values at each temperature as a function of $\sigma_{e}$. The highest $\Delta \sigma_{e}$ was seen at $4.2 \mathrm{~K}$ and the lowest is noted at $35 \mathrm{~K}$. The slopes of the curves at $25 \mathrm{~K}$ and $35 \mathrm{~K}$ are steeper than at $15 \mathrm{~K}, 8 \mathrm{~K}$ and $4.2 \mathrm{~K}$. A greater amount of initial continuous deformation corresponds to a steeper slope in these curves. As expected, temperature plays a significant role: the initiation strain for serrations is lower for lower temperatures. Furthermore, at $15 \mathrm{~K}$ and lower, where serrations begin at the yield point, the slope is the same (Fig. 2b), despite the specimen at $4.2 \mathrm{~K}$ and $8 \mathrm{~K}$ having consistently greater $\Delta \sigma_{e}$ than at $15 \mathrm{~K}$ over the course of deformation. These results indicate that the dislocation density, when serrations first appear, affects the $\Delta \sigma_{e}$ variation with deformation. A larger initial dislocation density, from continuous deformation, at $25 \mathrm{~K}$ and $35 \mathrm{~K}$ results in a stronger $\Delta \sigma_{e}-\sigma_{e}$ variation. 
However, at lower temperatures, where serrations begin at yield the $\Delta \sigma_{e}-\sigma_{e}$ variation is the same. The test at $4.2 \mathrm{~K}$ was performed in a machine with a higher stiffness. Taking this difference in stiffness into account, $\Delta \sigma_{e}$ is practically identical at $4.2 \mathrm{~K}$ and $8 \mathrm{~K}$. As the temperature decreases, the $\Delta \sigma_{e}$ increases (seen between $35 \mathrm{~K}$ and $8 \mathrm{~K}$ ), however, there is a plateauing of $\Delta \sigma_{e}$ below a certain temperature (seen between $8 \mathrm{~K}$ and $4.2 \mathrm{~K}$ ). The maximum plateau temperature is likely between $4.2-8 \mathrm{~K}$, since the change in $\Delta \sigma_{e}$ is negligibly low between theses temperatures.

Under the considerations of the thermomechanical effect, the $c_{p}$ and $\lambda$ of $\mathrm{CoCrFeMnNi}$ are sufficiently high to avoid localization of heat during deformation above $35 \mathrm{~K}$. Below $35 \mathrm{~K}$, heat localization becomes adequate to cause localized thermal softening. A lower temperature intensifies this effect since both $c_{p}$ and $\lambda$ exhibit a rapid, non-linear decrease in this temperature range [16]. Correspondingly $\Delta \sigma_{e}$ should increase more rapidly with decreasing temperature. However, the variation in $\Delta \sigma_{e}$ in the interval between $8 \mathrm{~K}$ and $4.2 \mathrm{~K}$ is negligibly low, contrary to the expectation of greatest difference of any interval seen. The thermomechanical effect, thus, seems unlikely under these circumstances.

Fig. 3 displays close-ups of stress vs. time curves for the experiments conducted at different temperatures under He vapor. The regions were chosen to illustrate approximately equal $\Delta \sigma_{e}$ at each temperature. With identical acquisition rates, stress drops appear to be of different speeds at different temperatures. The stress drop is faster at lower temperatures than at higher temperatures, taking $\sim 0.2 \mathrm{~s}$ for the stress drop at $15 \mathrm{~K}$ and $8 \mathrm{~K}, \sim 0.5 \mathrm{~s}$ for the drop at $25 \mathrm{~K}$ and $\sim 2 \mathrm{~s}$ at $35 \mathrm{~K}$ for a $\Delta \sigma_{e} \sim 50-60 \mathrm{MPa}$. However, the fastest portions of the stress drops constituting a majority of the total drop occurs in less than $0.05 \mathrm{~s}$ at $8 \mathrm{~K}$ and $15 \mathrm{~K}$. At $25 \mathrm{~K}$ and $35 \mathrm{~K}$, the shape of the curves is rather smooth. Thus, there is no distinct portion which exhibits extremely fast stress drops as in the previous two cases. Here, the stress drops usually require times in the order of $0.5 \mathrm{~s}$ and $2.5 \mathrm{~s}$, respectively. This yields useful information on the nature of stress drops: (i) the speed of the drop is temperature-dependent; (ii) the fastest portion of the drop is somewhat in the middle of the drop indicative of a critical condition which wears out. The latter point implies that upon reaching a critical condition there is a loss of dynamic equilibrium which accelerates into a stronger effect before finally slowing down under the influence of compensating factors. Under the mechanical model, a single dislocation source applies pressure on pileups in its vicinity leading to activation of multiple sources, which in turn do the same in quick succession. Subsequently the dislocations are blocked, likely by newly as well as previously formed locks. Under the thermomechanical model, an initial critical heat generated (greater than that which could have been efficiently dissipated) during localized deformation leads to greater deformation and corresponding heat. This would continue up to the point where the heat generated is suitably dissipated, slowing down the catastrophic process and achieving temperature equilibrium between the specimen and the cooling medium. Note that these considerations might be different for experiments conducted on single-crystalline materials (for example as in Ref. [33, 34]) where all individual, microscopic 

ultra-fast, distinct stress drops.
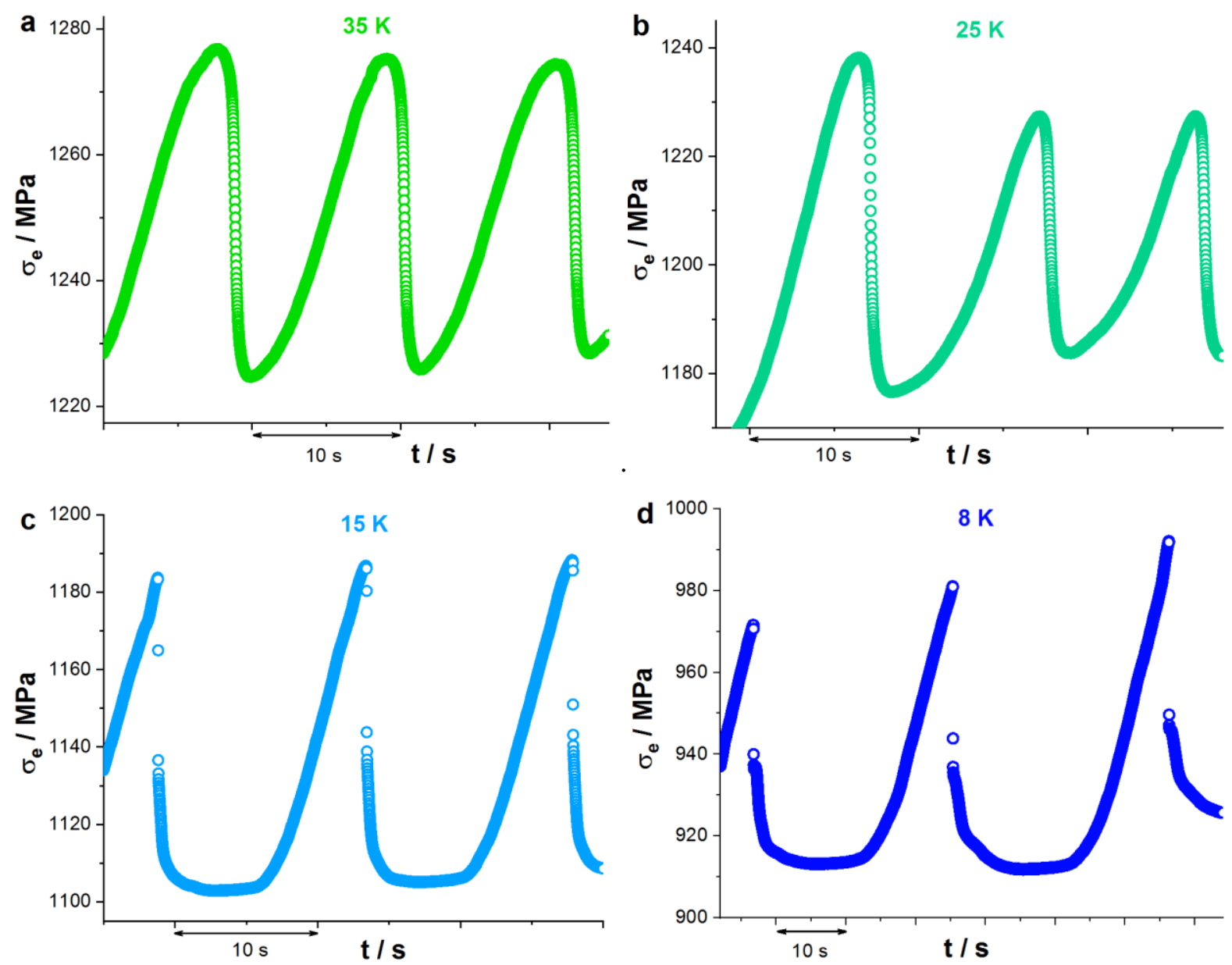

Figure 3: The close-ups of $\sigma_{e}-t$ curves of CoCrFeMnNi deformed at (a) $35 \mathrm{~K}$, (b) $25 \mathrm{~K}$, (c) $15 \mathrm{~K}$ and (d) $8 \mathrm{~K}$. In all of the illustrated cases $\Delta \sigma_{\mathrm{e}} \sim 50-60 \mathrm{MPa}$. The tests at $35 \mathrm{~K}$ and $25 \mathrm{~K}$ show a slower stress drop while those at $15 \mathrm{~K}$ and $8 \mathrm{~K}$ show a faster stress drop. It appears that in (c) and (d), the $\Delta \sigma_{\mathrm{e}}$ is greater than the specified 50-60 MPa. However, this stress drop is a combination of the specimen contribution as well as the machine contribution due to finite stiffness. Accordingly, the slower portion of the stress drop, seen after $70-80 \%$ of the drop is not considered when measuring $\Delta \sigma_{\mathrm{e}}$. This bottom portion is a result of the machine not being infinitely stiff and not being able to instantaneously accommodate the stress drop. An explanation on how maxima, minma and $\Delta \sigma_{\mathrm{e}}$ were determined, specifically in reletion to the specimen, can be found in the Appendix.

The rate of the stress drop in relation to temperature is likely associated with the higher dislocation velocity at very low temperatures. This jump in velocity is caused by the drop in viscous dampening of dislocations from the vanishing phonon scattering $[35,36]$. This is expected in metallic materials in general but additionally in alloys, this jump in velocity has an effect on the $\sigma_{Y S}-T$ trend. Dislocations are pinned to multiple solute atoms in an alloy. On application of an external mechanical stress, the dislocations overcome the pinning force of the various defects systematically and in succession. 
However, dislocations that achieve high velocities need only overcome a single pinning point. Once this is done, the momentum of this dislocation is so significant that the inertial effect alone overcomes the other pinning points. Thus, below a given threshold temperature the velocity of the dislocation increases rapidly and so does its ability to overcome solute pinning of dislocation. This manifests itself as a drop (dilute solid solutions) or plateau (concentrated solid solutions) of $\sigma_{\mathrm{YS}}$ at lower temperatures and is called the dynamic overshoot effect [37]. As the yield strength increases with decreasing temperature down to $15 \mathrm{~K}$ and plateaus out at even lower temperatures in the case of CoCrFeMnNi [15], it indicates that the dynamic overshoot effect and by extension the higher dislocation velocity is active at $T \leq 15 \mathrm{~K}$.

The tensile tests at different temperature reveal (i) a clear dependence of the serration behavior both on the temperature and the initial dislocation density and (ii) a variation in stress drop speed, likely due to the lack of viscous dampening of dislocations at very low temperatures.

\section{Influence of pre-deformation on serrations}

To verify the expected effect of initial dislocation density, tensile tests on pre-deformed specimens were performed at $8 \mathrm{~K}$. It has already been established that $\Delta \sigma_{e}$ is closely related to the engineering stress maxima [16] at a given temperature. When considering that the mechanical condition for instability has been met at a given temperature, a higher dislocation density should correspondingly amplify the stress drop amplitude. This would also be the expectation if dislocation-based plasticity leads to heating and correspondingly causes local thermal softening. Hence, a pre-deformed specimen should reflect the same trend as a fully recrystallized specimen, only joining it at a later stage in the curve with a much higher initial $\Delta \sigma_{e}$ (due to a higher $\sigma_{Y S}$ ).

In order to test this, a CoCrFeMnNi rod was wire-drawn to a true strain of $40 \%$ at $77 \mathrm{~K}$. Specimens were machined from it as stated in the experimental section and the tensile tests were carried out. The corresponding $\sigma_{e}-\varepsilon_{e}$ curve and $\Delta \sigma_{e}-\sigma_{e}$, are displayed in Fig. $4 \mathrm{a}$ and $\mathrm{b}$, respectively. The trends of $\Delta \sigma_{e}-\sigma_{e}$ variation are clearly similar. However, the slope in the case of the pre-deformed specimen is much steeper. Additionally, despite the expectedly higher initial dislocation density, the $\Delta \sigma_{e}$ is initially low but rises quickly. Although the phenomenon is dislocation-based, a higher dislocation density does not result in a distinctly higher $\Delta \sigma_{e}$, contrary to the expectations based on the proposed models of (i) Obst and Nyilas [21] and Seeger [26], (ii) Skoczeń et al. [30] and (iii) Basinski [18]. The reason for this will become apparent in the subsequent sections. 

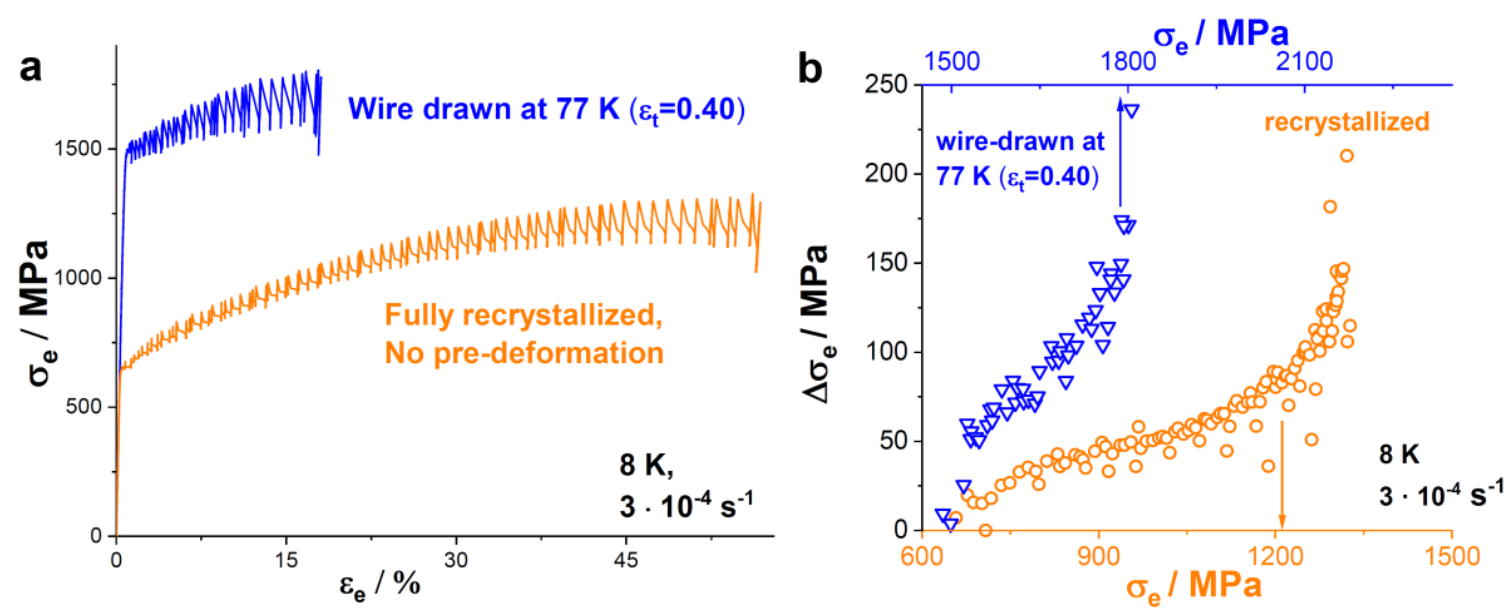

Figure 4: (a) $\sigma_{\mathrm{e}}-\varepsilon_{\mathrm{e}}$ curves of $\mathrm{CoCrFeMnNi}$ in the recrystallized and the pre-deformed condition deformed at $8 \mathrm{~K}$ and (b) corresponding $\Delta \sigma_{\mathrm{e}}-\sigma_{\mathrm{e}}$ curves. The range of the abscissa is the same in (b), implying that a visually steeper curve does have a higher $\Delta \sigma_{\mathrm{e}}-\sigma_{\mathrm{e}}$ variation.

\section{Possible alternate deformation mechanisms}

As the temperature decreases, the SFE changes in CoCrFeMnNi [38]. This has in turn an effect on the active deformation mechanisms. Fig. 5 shows true work-hardening rates plotted against true stress at four different temperatures for $\mathrm{CoCrFeMnNi}$. There is substantial overlap between all specimens, including the predominantly continuous $\sigma_{e}-\varepsilon_{e}$ curve of the specimen deformed at $35 \mathrm{~K}$ and the serrated curves at lower temperatures. The work-hardening rates of smooth and serrated portions of the specimen deformed at $25 \mathrm{~K}$ match up well and there is a consistent transition. Serrations are, thus, not associated with a change in the work-hardening rate. There is a general trend in the early portions of the work-hardening rate where it changes from a negative slope to a plateau, as a function of deformation. This is associated with the activation of deformation twinning and can also be seen at higher temperatures [39]. Generally, the activation of a new deformation mechanism is associated with a change in the work-hardening rate slope, as seen with $\mathrm{CoCrFeMnNi}$ and $\mathrm{CoCrNi}[15,16,40]$. However, it has already been confirmed through SEM and TEM analysis that the features seen in CoCrFeMnNi at $77 \mathrm{~K}$ [39] and at temperatures as low $4.2 \mathrm{~K}$ [16] are the same. This includes dislocation-based deformation and deformation twinning (there was no indication of $\varepsilon$-martensite formation at either temperature). So serrated plastic deformation at cryogenic temperatures is not caused by the activation of a new deformation mechanism. 


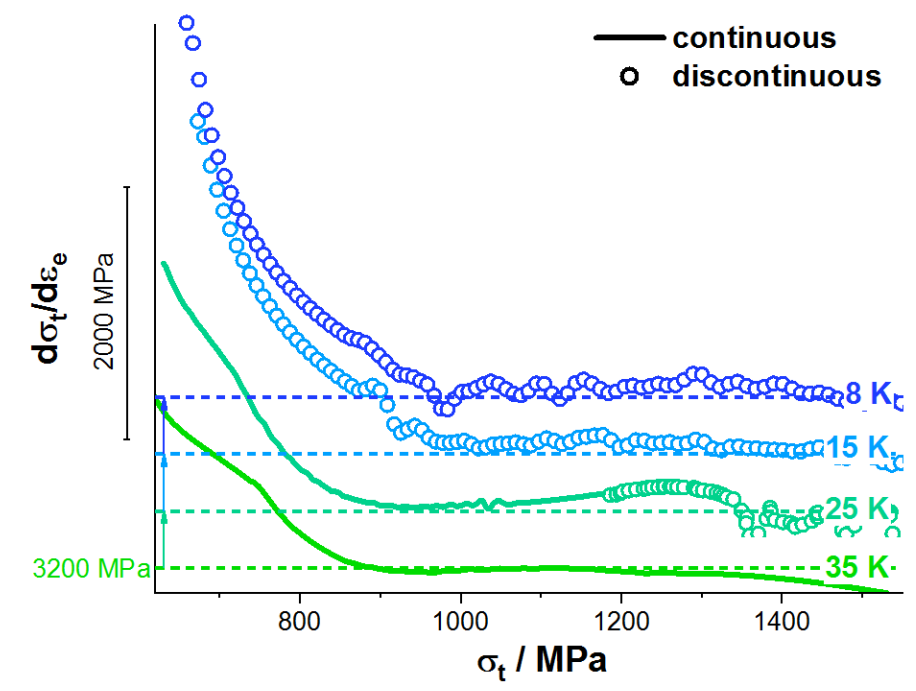

Figure 5: (a) $d \sigma_{t} / d \varepsilon_{t}-\sigma_{t}$ curves of CoCrFeMnNi deformed at $35 \mathrm{~K}, 25 \mathrm{~K}, 15 \mathrm{~K}$ and $8 \mathrm{~K}$. The plots have been smoothened to allow for interpretation of the serration data. The offset of each data set is indicated using the $3200 \mathrm{MPa}$ dashed baseline.

Based on these observations there is either (i) a characteristic phenomenon occurring only at very low temperatures or (ii) the boom of an already occurring phenomenon to facilitate this behavior. Dislocation density increases with deformation at a faster rate at lower temperatures [41], and this effect is maximized for alloys which have SFE in the same range as CoCrFeMnNi. As temperature decreases from $295 \mathrm{~K}$ to $4.2 \mathrm{~K}$, the dislocation density during deformation keeps increasing and the SFE decreasing [38]. In the same range of temperatures, the thermal vibrations become less influential and increase the effects of elastic distortion around defects: dislocation pinning as well as dislocationdislocation interaction. These changes result in a variation in (i) cross-slip ability, (ii) dislocation mobility of different types of dislocations, (iii) LC lock formation rate/influence and (iv) dislocation pile-up characteristics. We will address each of these aspects in the following section.

\section{Cross-slip propensity and the phenomenological model for serrated plastic deformation}

Cross-slip is associated with stage III deformation in single crystals but has also been documented at much lower macroscopic strains in polycrystalline samples. Cross-slip in metals and alloys is inherently more difficult at lower temperatures, as well as for lower SFE [42]. The activation energy originally proposed for cross-slip is related to the SFE $(\gamma)$ and the shear modulus $(G)$ as follows:

$$
E_{C S}=K \frac{G}{\gamma}
$$

Considering that $\gamma$ decreases and $G$ increases with decreasing temperature $E_{C S}$ varies inversely with temperature, becoming highest at $0 \mathrm{~K}$. This increasing $E_{C S}$ with decreasing $\gamma$ corresponds to the Schoeck-Seeger theory, as seen in the above equation [42]. However, even according to the widely accepted Friedel-Escaig model of cross-slip, as estimated by Duesbery et al. [43], a lower $\gamma$ and higher 
$G$ correspond to a higher $E_{C S}$. By contrast, the Fleischer model of cross-slip was not considered here, since it has mainly been used when discussing cross-slip in metals with high SFE (like Al) [44, 45]. The effect becomes pronounced by cross-slip being thermally activated with an Arrhenius type probability that strongly decreases with decreasing temperature.

Dislocation mobility of edge and screw dislocations should change minimally with temperature for the most part. However, screw dislocations in FCC metals and alloys are associated with an additional energy of motion at cryogenic temperatures. Jogged screw dislocations leave behind trails of vacancies on motion at cryogenic temperatures [34]. Jogged edge dislocations do not leave behind vacancies on gliding. Fig. 6a illustrates a scheme of an edge dislocation and a forest dislocation on two intersecting slip planes. Their intersection results in the jog formation of the edge dislocation, as seen in Fig. 6b. The direction of conservative motion of the original edge dislocation line as well as the jogged portion is the same. Fig. $6 \mathrm{c}$ and d show the same interaction between a screw dislocation and a forest dislocation. In this case, the direction of conservative slip is different for the original and jogged portion of the dislocation, respectively. The screw portion of the dislocation is pinned at the nodes connected to the jog, which is of edge character. On application of stress this screw portion bends as seen in Fig. 6e and beyond a certain point glides forward pulling the jog along with it. This results in non-conservative motion of the jog which leaves behind rows of vacancies (Fig. 6f). If multiple jogs exist, the dislocation can act as a Frank-Read (FR) source bowing out. The critical stress for this is given by [46]:

$$
\tau_{F R}=\frac{\alpha G b}{l_{0}}
$$

Here $\alpha$ is a coefficient associated with the FR mechanism, $b$ is the burgers vector and $l_{0}$ is the spacing between jogs. The equation for stress to move a jogged dislocation $\left(\tau_{j o g}\right)$, forming vacancies is the same, except the corresponding coefficient $\alpha$ is significantly lower $(\sim 0.1-0.2)$ than $\alpha_{F R}(\sim 1)$ [46]. The formation of vacancies during cryogenic deformation was verified experimentally [47, 48]. It should be noted that even though the current explanation only considers pure edge and pure screw dislocations, this explanation can be readily extended to near edge $60^{\circ}$ dislocations. Smith et al. [49] have already shown that many dislocations in a pile-up are of near edge nature in CoCrFeMnNi. A similar consideration was made in the past when studying low-temperature serrations in $316 \mathrm{LN}$ steel [21]. 

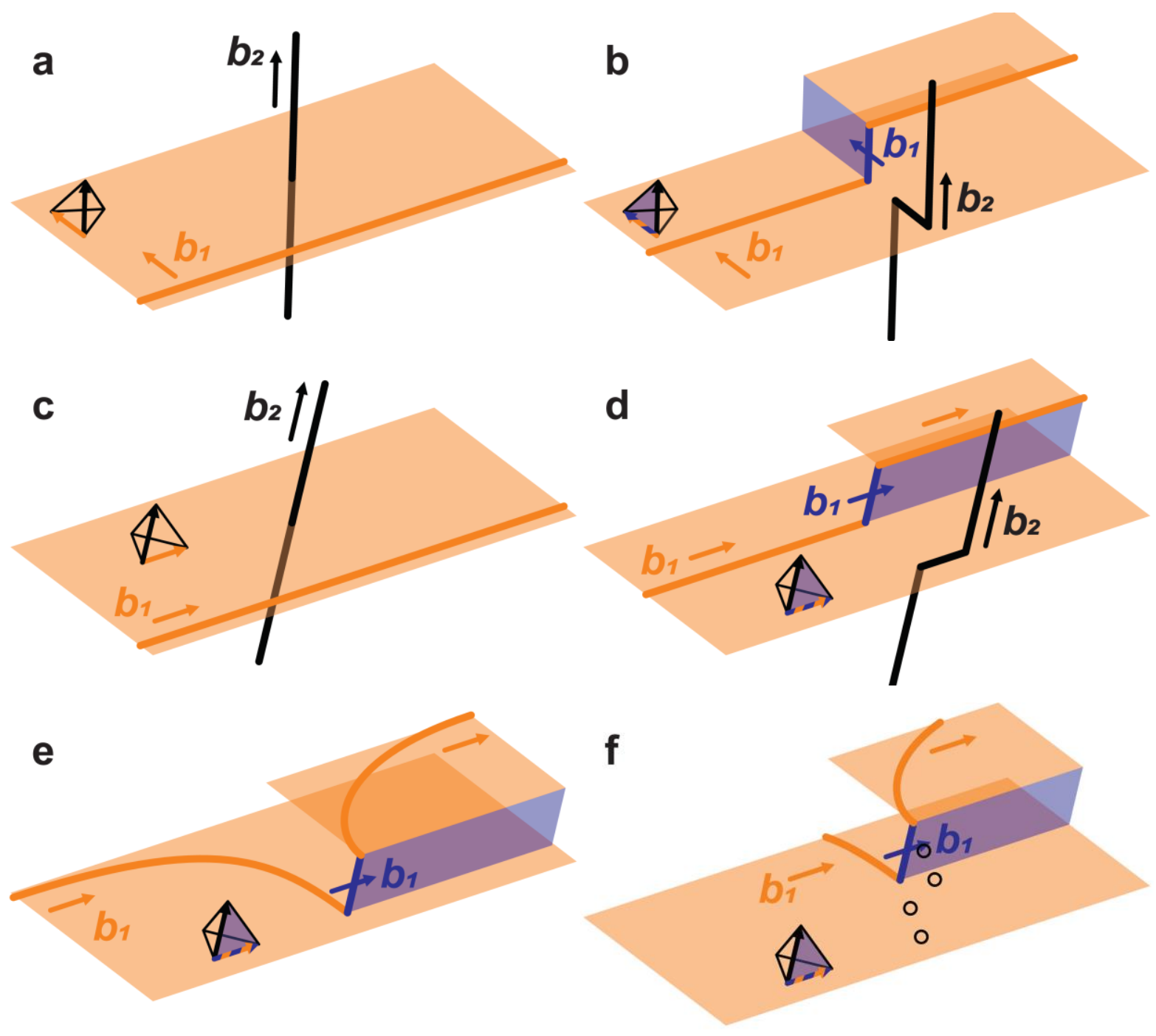

Figure 6: Intersection process of a moving dislocation with a forest screw dislocation: (a-b) moving edge and (c-f) moving screw dislocation. The slip planes of the original dislocations and the jogged segments are given by the orange and blue planes, respectively. The Thompson tetrahedra on the lower left, indicate the slip plane along which each of the dislocation can be found. Moving edge dislocation: (a) prior to intersection and (b) post-intersection with a glissile reaction segment. Moving screw dislocation: (c) prior to intersection, (d) post-intersection with sessile reaction segment, (e) bending of the main dislocation on either side of the jog and (f) formation of trails of vacancies (black circles) behind the jog as it engages in non-conservative motion.

As noted above, the SFE of CoCrFeMnNi decreases with decreasing temperature and, correspondingly, stacking fault width (SFW) increases. The interaction between solutes and dislocations is far more intense at cryogenic temperatures where distortion around a solute is severe [35] and the effect of solute dislocation interaction is intense in $\mathrm{CoCrFeMnNi}$ as well as $\mathrm{CoCrNi}$, as the temperature decreases [15]. This extends the SFW as leading and trailing Shockley partials are pinned by solutes and a wider SFW would increase the possibility of partials of two different slip systems interacting with each other. Also 
considering that there exists an inverse dependence of dislocation density on temperature, the number of LC locks at lower temperatures are likely higher.

Finally, with respect to the pile-up, the stress at the head of the pile up is dependent on the number of dislocations in the pile-up. Lower temperatures demand larger external stresses for dislocation motion as well as activation of dislocation sources. The equilibrium length of the pile-up increases with decreasing SFE and the equilibrium SFW of the dislocations in the pile-up becomes larger as well [50, $51,52]$. When the dislocation source is far from the LC lock, one may consider that for a given number of dislocations, due to the lower SFE at lower temperatures the pile-up length is larger. Alternatively considering a high dislocation density where the source-barrier spacing is low, the space in between can be completely filled up by a lower number of dislocations at lower temperatures. Additionally, considering the high dislocation density during deformation at lower temperatures the number of sources and locks on intersecting slip planes may be higher as well compared to higher temperatures. Thus, for both low and high barrier-source spacing the expected probability of dislocation interaction between dislocations of intersecting slip systems is higher at lower temperatures as a result of the lower SFE affecting the pile-up distance. The addition of dislocations to a pile-up continues until the stress at the head of the pile-up reaches the critical limit for breakdown and, as the number of dislocations in a pileup increases, the inter-dislocation spacing decreases [50, 51].

Following the above discussion, the origin for the serrated plastic flow is likely as follows: As the temperature decreases, SFE decreases and dislocation density increases for a given amount of strain. This results in an increase in the number of LC locks formed. During deformation the locks act as barriers to dislocations causing pile-ups, which further increase interactions between dislocations of different slip systems. Dislocations can escape when a sufficient number of them have piled up, equaling cross-slip stress at the head of the pile-up. Cross-slip is more favorable at higher temperatures and as temperature decreases the activation energy for cross-slip becomes higher making it even more difficult, owed to the decreasing contribution of thermal vibrations.

Additionally, jogged screw dislocations also generate vacancies on motion, making the stress required for screw dislocation motion higher at lower temperature. At some critical temperature, the cross-slip stress, as a result of higher cross-slip activation energy and higher stress for jogged screw dislocation motion, becomes competitive with the stress to activate dislocation source at the barrier (LC lock). Below this temperature, dislocation source activation at LC locks occurs, resulting in cooperative motion of dislocations as opposed to only cross-slip that still depends on thermal activation for each individual dislocation at the head of the pile-up. This is the key transition from continuous deformation to serrated deformation. Considering the gradual variation of cross-slip stress and stress to glide a jogged screw dislocation with temperature, a transition likely occurs at very low temperatures where edge dislocation motion becomes more important, and a greater proportion of pile-ups correspond in the activation of a dislocation source accompanied with sudden and massive dislocation proliferation as opposed to cross- 
slip of individual dislocations at the head of the pile-up. This is why a gradual change is seen between $35 \mathrm{~K}$ and $4.2 \mathrm{~K}$, with serrations beginning at low initial $\Delta \sigma_{e}$ each time. Dislocation density cannot be the only factor affecting serrations as they are mediated by cross-slip which is dependent on temperature of operation. Also, screw dislocations themselves are not less mobile but when jogged they become less mobile, as seen above. As deformation continues, screw dislocations intersect more forest dislocations effectively reducing $l_{0}$ between jogged portions making $\tau_{j o g}$ significantly higher. This makes screw dislocation motion significantly more difficult.

\section{Clarifications of former mechanical hypotheses}

Obst and Nyilas [21] had built on the mechanical model proposed in Ref. [26]. While the basis of this model was sound it has failed to sufficiently address two critical observations. (i) In Ref. [21], 316 LN steel was considered to have achieved criticality for serrated plastic flow at $34 \mathrm{~K}$ and lower, implying that screw dislocations were immobile below $34 \mathrm{~K}$. This does not explain why part of the deformation between $15 \mathrm{~K}$ and $34 \mathrm{~K}$ was continuous. (ii) As deformation proceeds the $\Delta \sigma_{e}$ increases in magnitude, presumably from the higher dislocation density. This should then imply that a higher initial dislocation density corresponds to a higher initial $\Delta \sigma_{e}$. This contradicts the current observations. However, based on the present model, both these issues can be resolved. (i) Cross-slip is active below $35 \mathrm{~K}$ and only decreases in propensity with the decreasing temperature. Accordingly, at $35 \mathrm{~K}$ and $25 \mathrm{~K}$, cross-slip is viable during the initial deformation, but as deformation continues, a significant number of dislocation intersections occur between mobile and forest dislocations. The free dislocation length between jogged portions $\left(l_{0}\right)$ correspondingly keeps decreasing, until a critical strain is achieved. Beyond this critical strain, a significant proportion of the screw dislocations have an $l_{0}$ too low to bow out and continue slipping. Subsequently, a portion of the dislocation-based plasticity has to be carried out through dislocation proliferation events at dislocation pile-ups. Thus the instability condition is met after an initial continuous deformation stage. (ii) In the case of the pre-deformed specimen, the propensity for cross-slip should be similar to that of the fully recrystallized specimen, considering the temperature of deformation is similar. The only difference is a higher dislocation density and larger number of jogged dislocations. However, since the yield stress is so much higher in this case, based on Eq. 2, cross-slip can be activated for several dislocations of smaller $l_{0}$ than in the fully recrystallized condition. Due to these factors the plasticity in the initial stages is still sufficiently mediated by cross-slip resulting in a relatively low stress drop amplitude. Nevertheless, the high dislocation density results in a rapid change in the availability of dislocation segments with sufficiently large $l_{0}$ for screw dislocation motion and cross-slip. The proportion of dislocation proliferation events, thus, increases rapidly and correspondingly the $\Delta \sigma_{e}-\sigma_{e}$ trend is significantly steeper than in the fully recrystallized condition.

Skoczeń et al. [30] have considered an exponential increase in the number of dislocation pile-up groups, presumably blocked by LC locks. This was applied to model the serration behavior seen in $316 \mathrm{LN}$ steel, based on their own experimental results as well as the work in Ref. [21]. The LC lock density and 
dislocation density were considered to be interdependent. There was also an implied exponential increase in the number of LC locks below $40 \mathrm{~K}$. While this explanation suitably covers the observations of the current results for CoCrFeMnNi between $35 \mathrm{~K}$ and $8 \mathrm{~K}$ it does not have a phenomenological explanation, i.e. an appropriate basis for an exponential increase in LC locks below $40 \mathrm{~K}$. However, if one considers pile-ups where dislocations cannot cross-slip, their numbers may grow rapidly, as transition takes place from cross-slip to dislocation proliferation events. In this way, the need for a boom in LC lock density is circumnavigated. This is more plausible considering that: (i) An inverse exponential relationship between temperature and LC lock density would require the largest difference to be seen between $8 \mathrm{~K}$ and $4.2 \mathrm{~K}$ which have almost identical $\Delta \sigma_{\mathrm{e}}$; (ii) An exponential increase in LC lock density should correspond to a noticeable jump in the work-hardening rate as the temperature decreases however, this was not the case; (iii) There appears to be no phenomenological justification of LC lock density boom in the temperature interval of $4.2-40 \mathrm{~K}$. Thus, the increase in number of dislocation pile-ups that will result in dislocation proliferation events likely increases, but it is not exponential in nature and is not due to a rapid increase in the number of LC locks.

\section{Influence of deformation twinning and $\varepsilon$-martensite formation on serrations}

Low temperature tensile tests were conducted on $\mathrm{CoCrNi}$ and $\mathrm{CoNi}$ in the same way as on $\mathrm{CoCrFeMnNi}$.

The tests were conducted at $8 \mathrm{~K}$. Fig. 7a shows the $\sigma_{e}-\varepsilon_{e}$ curves and Fig. $7 \mathrm{~b}$ shows the corresponding $\Delta \sigma_{e}-\sigma_{e}$ curve. CoCrNi shows large stress drops just as with CoCrFeMnNi. CoNi, however, shows much smaller stress drops after significant deformation.
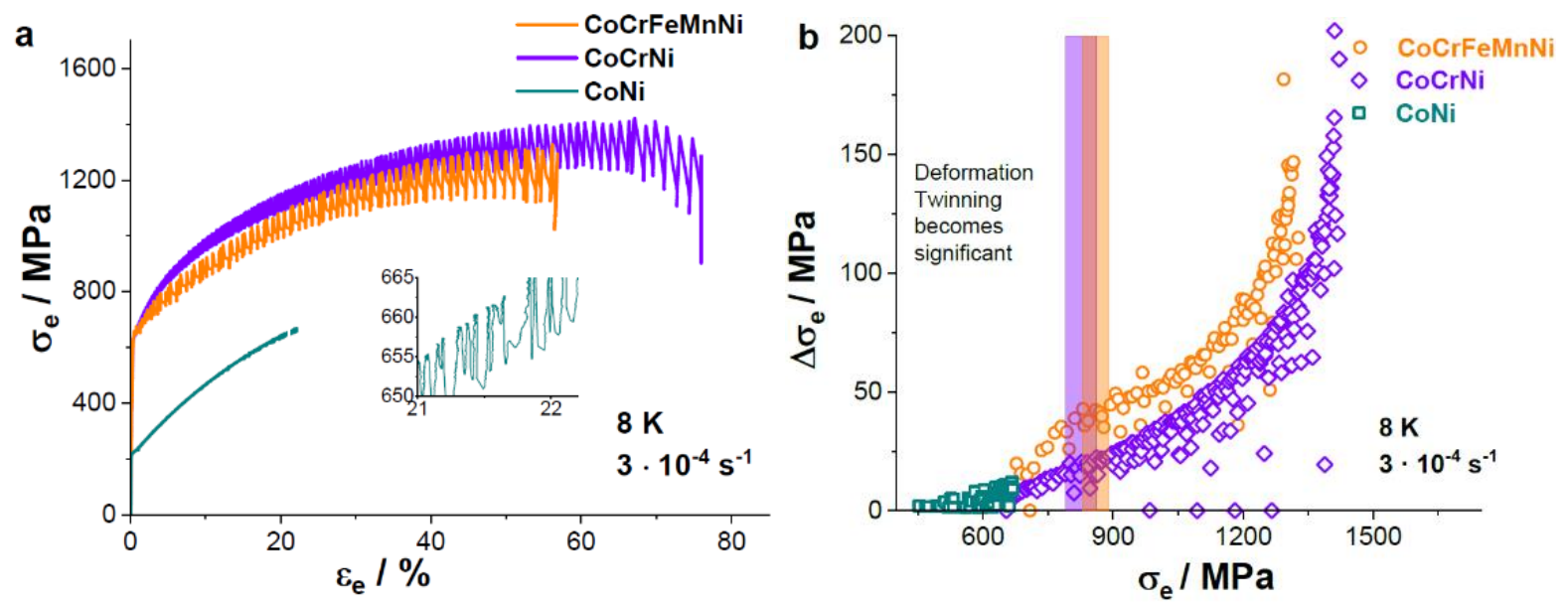

Figure 7: (a) $\sigma_{e}-\varepsilon_{e}$ curves of $\mathrm{CoCrFeMnNi}, \mathrm{CoCrNi}$ and $\mathrm{CoNi}$ deformed at $8 \mathrm{~K}$, (b) corresponding $\Delta \sigma_{e}-\sigma_{e}$ curves. The inset in (a) shows the serrated behavior seen in CoNi at the later stages of deformation. The colored columns in (b) indicate the minimum $\sigma_{e}$ for initiation of deformation twinning (orange for $\mathrm{CoCrFeMnNi}$ and purple for $\mathrm{CoCrNi}$ ). $\sigma_{e}$ was determined based on the change in the workhardening rate indicating activation of a new deformation mechanism, namely deformation twinning. A detailed discussion of deformation mechanisms and microstructural evolution can be found in Ref. [15]. 
The $\Delta \sigma_{e}-\sigma_{e}$ analysis illustrates several points. Firstly, the $\sigma_{e}$ maxima for $\mathrm{CoCrNi}$ are higher than for the $\mathrm{CoCrFeMnNi}$, but $\Delta \sigma_{e}$ is not correspondingly higher. $\Delta \sigma_{e}$ is, thus, not only stress dependent but it is also material dependent. This material dependence is possibly related to (i) SFE and/or (ii) solutedislocation interaction.

SFE inherently dictates serration flow as discussed in the previous Section. It essentially begins below the temperature where cross-slip stress becomes comparable to critical stress for dislocation source activation at LC locks [26]. Cross-slip is severely dependent on SFE, with Shockley partials having to constrict into a full dislocation before cross slipping onto an intersecting slip plane. A lower SFE implies a higher temperature of activated serrated plastic deformation. However, the evidence does not bear this out completely. $316 \mathrm{LN}$ steel, despite having a lower room temperature SFE than CoCrFeMnNi, shows very similar temperatures of serrated plastic flow (the SFE of similar composition steels are $\gamma \leq$ $20 \mathrm{~mJ} \cdot \mathrm{m}^{-2}[53,54]$, while $\mathrm{CoCrFeMnNi}$ possesses $\gamma \sim 30 \mathrm{~mJ} \cdot \mathrm{m}^{-2}$ [55]). CoCrFeMnNi also shows slightly higher temperatures of (i) serrations being observed and (ii) serrations observed at yield point. Therefore, it is more likely that SFE of a certain range manifests serrated plastic flow at a given temperature. A direct correlation between equilibrium SFE and propensity for cryogenic serrated plastic deformation, as expected from previous literature [26, 46], is inappropriate.

Solute influence is another key point that could be brought into play. In general, solute content positively correlates with the temperature of serrated plastic flow [22]. Quantitative $\Delta \sigma_{e}-$ solute correlations have not previously been studied, though, it is qualitatively apparent that a higher solute content is favorable for serrated plastic flow. Greater solute content corresponds to greater level of solid solution strengthening. A possible connection is that the larger amplitudes are a result of higher strengths of alloys with greater solute content. However, this is not the case in the current set of results where $\Delta \sigma_{e}$ is lower for $\mathrm{CoCrNi}$ than for $\mathrm{CoCrFeMnNi}$, despite the slightly higher $\sigma_{e}$ maximum of $\mathrm{CoCrNi}$. In the current case, solutes may interact with leading and trailing partials, pinning them and correspondingly increase the scatter of SFW around the expected value as stated in the previous Section. Even at higher temperatures, complex alloys have a larger scatter of observed SFWs while pure metals have consistent SFW and correspondingly consistent SFE. This is especially true in the case of CoCrFeMnNi [49]. At temperatures close to $0 \mathrm{~K}$, the thermal vibrations are insignificant and correspondingly the interactions of dislocations between one another and solutes is based primarily on the attraction and repulsion due to elastic distortion around the respective defects. It is, thus, possible for solutes to indirectly affect the SFW of dislocations which would have an effect on (i) energy required to constrict partials, (ii) dislocation spacing in pile-ups and (iii) possibly LC lock formation. All three factors directly affect serration behavior. The current set of alloys is not ideal to make quantitative statements on the effect of solutes on serration behavior but it is clear that concentrated solid solutions which have an inherently severe solid solution strengthening effect show intense serration behavior ( $\mathrm{CoCrNi}$ and $\mathrm{CoCrFeMnNi}$ ), as opposed to weakly solid solution strengthened alloys (CoNi, despite the high solute content). 
The deformation mechanisms in CoCrFeMnNi at $8 \mathrm{~K}$ were previously discussed in Ref. [15]. $\mathrm{CoCrFeMnNi}$ and CoCrNi show intense deformation twinning while CoNi exhibits fairly insignificant twinning. This is a result of the different SFE of the alloys. An additional effect of the SFE is the appearance of $\varepsilon$-martensite in $\mathrm{CoCrNi}$ at $8 \mathrm{~K}$ [15]. The $\varepsilon$-martensite can be detected in the form of a twin-martensite nano-laminate on practically all twin boundaries. The deformation twinning in $\mathrm{CoCrFeMnNi}$ and $\mathrm{CoCrNi}$ is associated with a minimum stress required to activate it [39, 40]. Correspondingly, this is achieved only after the tensile specimen undergoes a minimum amount of $\varepsilon_{e}$ ranging between $6-10 \%[15,16]$. Based on the mechanism of formation of $\varepsilon$-martensite in $\mathrm{CoCrNi}$, a leading partial dislocation interacts with a twin boundary [56] to create $\varepsilon$-martensite sandwiched between matrix and twinned regions. This must take place after the initial $6-10 \% \varepsilon_{e}$ as well. The estimated stress for deformation twinning in $\mathrm{CoCrFeMnNi}$ and $\mathrm{CoCrNi}$ are marked by the colored columns in Fig. 7b. It is evident that the $\Delta \sigma_{e}-\sigma_{e}$ trend is consistent in both alloys below and above the minimum twin stresses with no abrupt jumps or trend variations. It should be noted that serrations were observed starting at the yield point in both alloys as well as subsequently in CoNi. Evidently, deformation twinning and the formation of $\varepsilon$-martensite are not necessary for the appearance of serrations. This is contrary to plastic instability at other temperatures showing abrupt and distinct transitions with the activation of TRIP/TWIP effects [57, 28, 58, 29].

Deformation twinning and $\varepsilon$-martensite have an associated heat of deformation. This heat is distinctly greater than that for purely dislocation-based deformation [59]. In the case of a thermomechanical effect influencing the $\Delta \sigma_{e}$, there would have been a clear change in its value at the initial twin stress. At this stage the extent of deformation twinning is already sufficient to cause a substantial change in the workhardening rate of the alloy. The energy released through deformation twinning is likely sufficiently high to show a $\Delta \sigma_{e}$ variation, either as a jump in amplitude or a change in slope of the $\Delta \sigma_{e}-\sigma_{e}$ trend (when considering the thermomechanical model). Moreover, $\mathrm{CoCrNi}$ shows the formation of $\varepsilon$-martensite layers on practically all twin boundaries when deformed at $8 \mathrm{~K}$ [15]. This additional and generous martensite formation should have further increased heat generation in $\mathrm{CoCrNi}$ as compared to $\mathrm{CoCrFeMnNi}$. However, the $\Delta \sigma_{e}-\sigma_{e}$ trend in both alloys is similar and CoCrNi consistently maintains a lower $\Delta \sigma_{e}$. This is, once again, contrary to the predictions of the thermomechanical model and thus, further invalidates it.

\section{Conclusions}

Following conclusions are drawn from the results and the discussion presented in this article:

1. CoCrFeMnNi is a strongly solid solution strengthened, stable single-phase FCC alloy, of appropriate SFE, correspondingly acting as the ideal candidate to study the nature and aspects of low temperature serrated plastic flow.

2. Both temperature of operation and initial dislocation density strongly influence the stress drop amplitude variation and are both factors affecting the instability condition. 
3. The current article puts forth a comprehensive phenomenological model on low temperature serrations based on inability of dislocations to cross-slip and the appearance of dislocation proliferation events. This is seen as a gradual transition from non-serrated to serrated deformation at lower temperatures, as opposed to the conventional model which considers a single discrete transition.

4. Neither deformation twinning nor $\varepsilon$-martensite formation are critical for serration behavior, they additionally do not have any measureable influence on the serrated plastic flow.

5. The thermomechanical model of serrations contradicts several of the observations in the current experiments and is thus invalid as a cause for stress drops.

The current results indicate a strong link between dislocation-solute interaction during deformation and a corresponding effect on dislocation constriction and cross-slip dynamics. Serrated plastic behavior could thus be ideal for evaluation of SFW, constriction and cross-slip potential in materials based on solute-dislocation interaction during deformation, as opposed to equilibrium SFE estimations.

\section{Data availability statement}

The raw data required to reproduce these findings are available on request to alexander.kauffmann@kit.edu. The processed data required to reproduce these findings are available on request to alexander.kauffmann@ kit.edu.

\section{Prime novelty statement}

We confirm that this manuscript has not been published previously by any of the authors and is not under consideration for publication in another journal.

\section{Declaration of competing interest}

The authors declare that they have no known competing financial interests or personal relationships that could have appeared to influence the work reported in this paper.

\section{Authorship contribution statement}

A.S. Tirunilai: Conceptualization, Methodology, Investigation, Writing - original draft, Visualization. T. Hanemann: Investigation, Writing - review \& editing. K.-P. Weiss: Methodology, Investigation, Writing - review \& editing. J. Freudenberger: Methodology, Investigation, Writing - review \& editing. M. Heilmaier: Resources, Writing - review \& editing, Supervision. A. Kauffmann: Conceptualization, Methodology, Investigation, Resources, Writing - original draft, Visualization, Supervision, Funding acquisition.

\section{Acknowledgements}

Financial support by the Deutsche Forschungsgemeinschaft within the framework of the Priority Program "Compositionally Complex Alloys - High-Entropy Alloys (CCA-HEA)" (SPP 2006) is 
488 gratefully acknowledged, grants no. KA 4631/1-1 and WE 6279/1-1. We gratefully acknowledge the 489 experimental support of $\mathrm{V}$. Tschan. 


\section{Appendix}
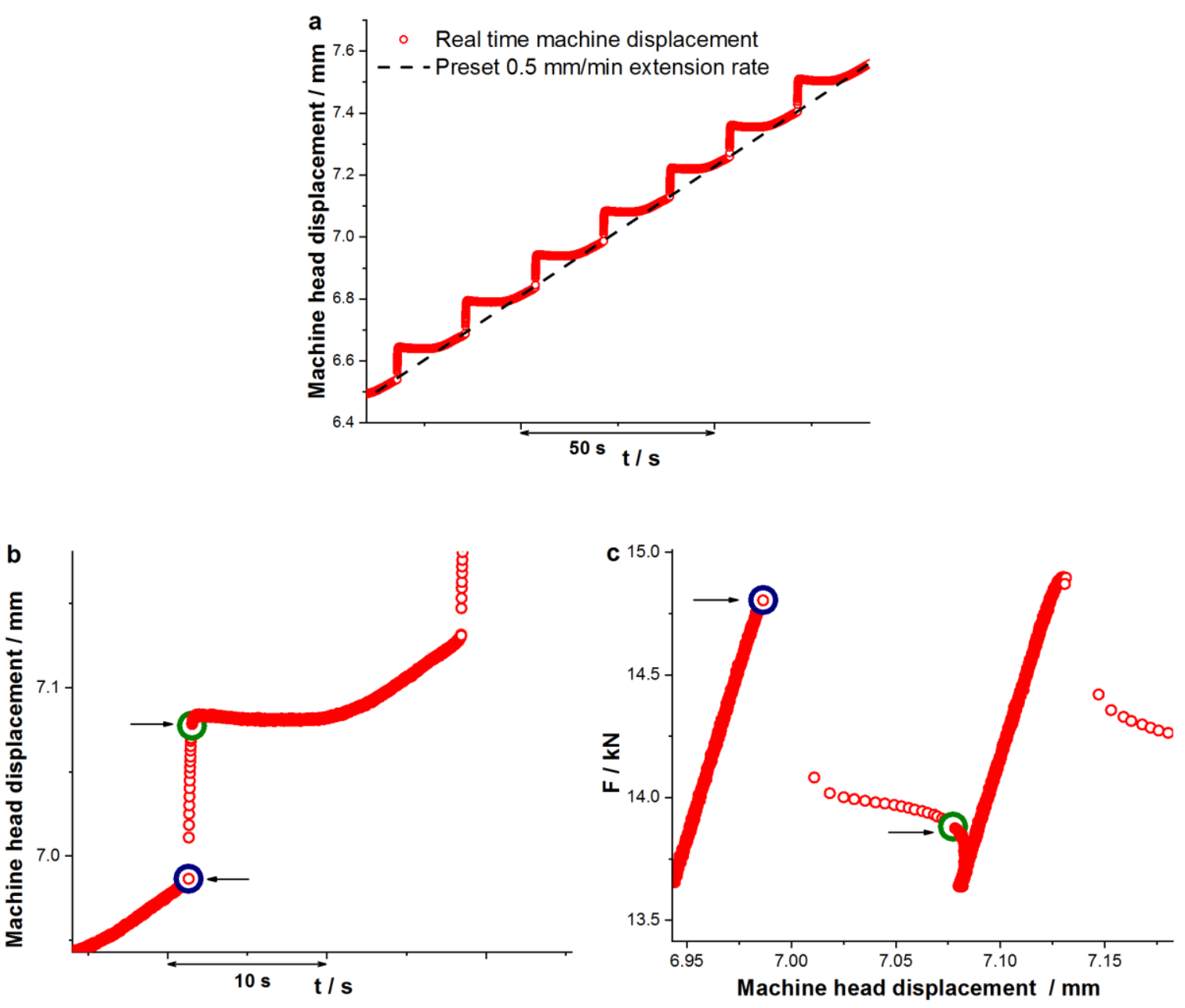

Figure 8: (a) Machine displacement vs. time, (b) close up of machine displacement vs. time and corresponding (c) force vs. machine displacement close up. Points marked in blue indicate local maxima while those marked in green indicate local minima. Both maxima and minima points are indicated by arrows as well. For details how these extreme were evaluated, please refer to the text.

Fig. 8(a) illustrates the variation of machine displacement as a function of time and Fig. 8(b) is a closeup of the same. In the case of continuous deformation, the expectation is that of a positive linear variation of machine displacement with time, equaling the extension rate of the machine cross-head (marked by the black dashed line in Fig. 8(a)). Since the test is displacement controlled, and the test operates under a preset extension rate a sudden stress drop would ideally be accommodated by a corresponding drop in the force applied by the machine to maintain the constant extension rate. However, for a sudden drop in stress (like in the case of a serration), due to limited stiffness of the machine and a finite reaction time, there is no instantaneous drop in machine force to maintain the same extension rate. This results in a sudden rapid extension event, as seen in Fig. 8(b), since the applied force has not been lowered sufficiently yet, to accommodate the preset extension rate. Quickly, thereafter the applied force by the machine is lowered. However, this drop in force is greater than the drop in force corresponding to stress drop of the specimen. So only a part of the total observed drop in force is a result of contribution form 
the specimen. To identify the point of transition from drop in force due to specimen to drop in force due to machine overcompensation, the machine displacement is examined. Essentially, since there is a greater drop in applied force than needed to cause plastic deformation in the specimen, the specimen is currently loaded below its elastic limit. Furthermore, the local machine displacement rate is higher than the preset $0.5 \mathrm{~mm} / \mathrm{min}$. This is accommodated by ensuring no further change in cross-head displacement. This can be seen as the plateau portion in Fig. 8(b) following the jump. This continues till the local extension rate is equal to the preset. This is clear from Fig. 8(a) where the dotted line representing the preset appears as the average of the step like serrated behavior. Based on the above sequence of events during a serration, there is a stress drop for the specimen followed by an additional stress drop contribution form the machine. The latter part provides no additional machine displacement. During the actual stress drop that is caused by the specimen there is a jump in the machine displacement. Therefore, the start of the serration is marked by the point immediately preceding the jump in machine displacement and the end is marked by the point immediately preceding the plateau of the machine displacement. These points are marked in Fig. 8(b) and correspondingly on the stress strain diagrams on Fig. $8(\mathrm{c})$. The stress maxima and minima determined by these methods are significantly more accurate than measurements of absolute maxima and minima of a given serration. This makes comparison between serrations under different experimental conditions plausible. In the case of test conducted in the ATLAS machine at $4.2 \mathrm{~K}$ the minima was taken as the absolute value. In this case the machine stiffness was extremely high making machine contribution to the stress drop practically negligible.

\section{References}

[1] B. S. Murty, J. W. Yeh and S. Ranganathan, High-Entropy Alloys, Amsterdam: Elsevier, 2014.

[2] J. W. Yeh, Y. L. Chen, S. J. Lin and S. K. Chen, "High-Entropy Alloys - A New Era of Exploitation," Materials Science Forum, vol. 560, pp. 1 - 9, 2007.

[3] M. H. Tsai, "Physical Properties of High Entropy Alloys," Entropy, vol. 15, pp. 5338 - 5345, 2013.

[4] O. N. Senkov, J. M. Scott, S. V. Senkova, D. B. Miracle and C. Woodward, "Microstructure and room temperature properties of a high-entropy TaNbHfZrTi alloy," Journal of Alloys and Compounds, vol. 509, pp. 6043 - 6048, 2011.

[5] L. Lilensten, J. P. Couzinié, L. Perrière, A. Hocini, C. Keller, G. Dirras and I. Guillot, "Study of a bcc multi-principal element alloy: Tensile and simple shear properties and underlying deformation mechanisms," Acta Materialia, vol. 142, pp. 131 - 141, 2018.

[6] C. Varvenne, A. Luque and W. A. Curtin, "Theory of strengthening in fcc high entropy alloys," Acta Materialia, vol. 118, pp. 164 - 176, 2016.

[7] Z. Wu, H. Bei, F. Otto, G. M. Pharr and E. P. George, "Recovery, recrystallization, grain growth and phase stability of a family of FCC-structured multi-component equiatomic solid solution alloys," Intermetallics, vol. 46, pp. 131 - 140, 2014. 
[8] J. Yeh, "Physical Metallurgy of High-Entropy Alloys," The Journal of The Minerals, Metals \& Materials Society, vol. 67, no. 10, pp. 2254 - 2261, 2015.

[9] B. Cantor, I. T. H. Chang, P. Knight and A. J. B. Vincent, "Microstructural development in equiatomic multicomponent alloys," Materials Science and Engineering A, Vols. 375 - 377, pp. $213-218,2004$.

[10] F. Otto, A. Dlouhý, C. Somsen, H. Bei, G. Eggeler and E. P. George, "The influences of temperature and microstructure on the tensile properties of a CoCrFeMnNi high-entropy alloy," Acta Materialia, vol. 61, pp. 5743 - 5755, 2013.

[11] Z. Wu, H. Bei, G. Pharr and E. P. George, "Temperature dependence of the mechanical properties of equiatomic solid solution alloys with face-centered cubic crystal structures," Acta Materialia, vol. 81, pp. 428 - 441, 2014.

[12] F. Otto, N. L. Hanold and E. P. George, "Microstructural evolution after thermomechanical processing in an equiatomic, single-phase CoCrFeMnNi high-entropy alloy with special focus on twin boundaries," Intermetallics, vol. 54, pp. 39 - 48, 2014.

[13] S.-H. Joo, H. Kato, M. J. Jang, J. Moon, C. W. Tsai, J. W. Yeh and H. Kim, "Tensile deformation behavior and deformation twinning of an equimolar CoCrFeMnNi high-entropy alloy," Materials Science \& Engineering A, vol. 689, pp. 122 - 133, 2017.

[14] A. J. Zaddach, C. Niu, C. C. Koch and D. L. Irving, "Mechanical Properties and Stacking Fault Energies of NiFeCrCoMn High-Entropy Alloy," JOM, vol. 65, pp. 1780 - 1789, 2013.

[15] A. S. Tirunilai, T. Hanemann, C. Reinhart, V. Tschan, K.-P. Weiss, G. Laplanche, J. Freudenberger, M. Heilmaier and A. Kauffmann, "Comparison of cryogenic deformation of the concentrated solid solutions CoCrFeMnNi, CoCrNi and CoNi," Materials Science and Engineering: A, vol. 783, 2020.

[16] A. S. Tirunilai, J. Sas, K.-P. Weiss, H. Chen, D. V. Szabó, S. Schlabach, S. Haas, D. Geissler, J. Freudenberger, M. Heilmaier and A. Kauffmann, "Peculiarities of deformation of CoCrFeMnNi at cryogenic temperatures," Journal of Materials Research, vol. 33, no. 19, pp. 3287 - 3300, 2018.

[17] M. Naeem, H. He, F. Zhang, H. Huang, S. Harjo, T. Kawasaki, B. Wang, S. Lan, Z. Wu, F. Wang, Y. Wu, Z. Lu, Z. Zhang, C. T. Liu and X.-L. Wang, "Cooperative deformation in high-entropy alloys at ultralow temperatures," Science Advances, vol. 6, no. 13, 2020.

[18] Z. S. Basinski, "The instability of plastic flow of metals at very low temperatures," Proceedings of the Royal Society A, vol. 240, no. 1221, pp. 354 - 358, 1957.

[19] T. H. Blewitt, R. R. Coltman and J. K. Redman, "Low-Temperature Deformation of Copper Single Crystals," Journal of Applied Physics, vol. 28, pp. 651 - 661, 1957.

[20] V. A. Moskalenko, V. D. Natsik and V. N. Kovaleva, "Low temperature anomalies of Ti plasticity resulting from inertial properties of dislocation motion," Materials Science and Engineering: $A$, Vols. $309-310$, pp. $173-177,2001$. 
[21] B. Obst and A. Nyilas, "Experimental evidence on the dislocation mechanism of serrated yielding in f.c.c. metals and alloys at low temperatures," Materials Science and Engineering A, vol. 137, pp. 141 - 151, 1991.

[22] V. V. Pustovalov, "Serrated deformation of metals and alloys at low temperatures (Review)," Low Temperature Physics, vol. 34, pp. 683 - 723, 2008.

[23] K. Jin, S. Mu, K. An, W. D. Porter, G. D. Samolyuk, G. M. Stocks and H. Bei, "Thermophysical properties of Ni-containing single-phase concentrated solid solution alloys," Materials and Design, vol. 117, pp. 185 - 192, 2017.

[24] K. A. Dahmen, Y. Ben-Zion and J. T. Uhl, "Micromechanical Model for Deformation in Solids with Universal Predictions for Stress-Strain Curves and Slip Avalanches," Physical Review Letters, vol. 102, 2009.

[25] Y. Ben-Zion, "Statistics of Earthquakes in Simple Models of Heterogeneous Faults," Physical Review Letters, vol. 78, no. 25, pp. 4885 - 4888, 1997.

[26] A. Seeger, "The mechanism of glide and work hardening in Face-Centered Cubic and HexagonalClose Packed metals," in Dislocations and Mechanical Properties of Crystals, Acta Crystallographica 11 (4), 1958, pp. 243 - 330.

[27] J. P. Hirth and J. Lothe, "Glide of jogged dislocations," in Theory of dislocations, New York, St. Louis, San Francisco, Toronto, London, Sydney, McGraw-Hill Book Company, 1968, pp. 535 556.

[28] T. A. Lebedkina, M. A. Lebyodkin, J.-P. Chateau, A. Jacques and S. Allain, "On the mechanism of unstable plastic flow in an austenitic FeMnC TWIP steel," Materials Science and Engineering A, vol. 519, pp. 147 - 154, 2009.

[29] A. Kozłowska, B. Grzegorczyk, M. Morawiec and A. Grajcar, "Explanation of the PLC Effect in Advanced High-Strength Medium-Mn Steels. A Review," Materials, vol. 12, no. 24, 2019.

[30] B. Skoczeń, J. Bielski, S. Sgobba and D. Marcinek, "Constitutive model of discontinuous plastic flow at cryogenic temperatures," International Journal of Plasticity, vol. 26, pp. 1659 - 1679, 2010.

[31] J. Tabin, B. Skoczen and J. Bielski, "Strain localization during discontinuous plastic flow at extremely low temperatures," International Journal of Solids and Structures, Vols. 97 - 98 , pp. $593-612,2016$.

[32] J. Sas, K.-P. Weiss and N. Bagrets, "Cryomak- the overview of cryogenic testing facilities in Karlsruhe," Acta Metallurgica Slovaca, vol. 21, no. 4, pp. 330 - 338, 2015.

[33] A. M. Dolgin and V. Z. Bengus, "Kinetics of high-velocity processes of low temperature jump-like deformation of niobium," physica status solidi (a), vol. 94, no. 2, pp. 529 - 535, 1986.

[34] V. S. Bobrov and M. Lebyodkin, "Electrical Effects in Low-Temperature Abrupt Ddeformation of Al (as translated to English in Sov. Phys. Solid State vol. 31, no.6, pp. 982 - 985, 1989, https://www.researchgate.net/publication/237313144)," Fizika Tverdogo Tela, vol. 31, no. 6, pp. 120 - 126, 1989. 
[35] R. B. Schwarz, R. D. Isaac and A. V. Granato, "Dislocation Inertial Effects in the Plastic Deformation of Dilute Alloys of Lead and Copper," Physical Review Letters, vol. 38, no. 10, pp. 554 - 557, 1977.

[36] R. B. Schwarz and R. Labusch, "Dynamic simulation of solution hardening," Journal of Applied Physics, vol. 49, pp. 5174 - 5187, 1978.

[37] A. S. Argon, Strengthening Mechanisms in Crystal Plasticity, New York: Oxford University Press, 2008.

[38] S. Huang, W. Li, S. Lu, F. Tian, J. Shen, E. Holmström and L. Vitos, "Temperature dependent stacking fault energy of FeCrCoNiMn high entropy alloy," Scripta Materialia, vol. 108, pp. 44 47, 2015.

[39] G. Laplanche, A. Kostka, O. M. Horst, G. Eggeler and E. P. George, "Microstructure evolution and critical stress for twinning in the CrMnFeCoNi high-entropy alloy," Acta Materialia, vol. 118, pp. 152 - 163, 2016.

[40] G. Laplanche, A. Kostka, C. Reinhart, J. Hunfeld, G. Eggeler and E. P. George, "Reasons for the superior mechanical properties of medium-entropy $\mathrm{CrCoNi}$ compared to high-entropy CrMnFeCoNi," Acta Materialia, vol. 128, pp. 292 - 303, 2017.

[41] V. Subramanya Sarma, J. Wang, W. W. Jian, A. Kauffmann, H. Conrad, J. Freudenberger and Y. T. Zhu, "Role of stacking fault energy in strengthening due to cryo-deformation of FCC metals," Materials Science and Engineering A, vol. 527, pp. 7624 - 7630, 2010.

[42] P. Haasen, "Plastic deformation of nickel single crystals at low temperatures," The Philosophical Magazine: A Journal of Theoretical Experimental and Applied Physics, vol. 3, no. 28, pp. 344 418, 1958.

[43] M. S. Duesbery, N. P. Louat and K. Sadananda, "The mechanics and energetics of cross-slip," Acta Metallurgica et Materialia, vol. 40, no. 1, pp. 149 - 158, 1992.

[44] C. Jin, Y. Xiang and G. Lu, "Dislocation cross-slip mechanisms in aluminum," Philosophical Magazine, vol. 91, no. 32, pp. 4109 - 4125, 2011.

[45] J. Marian, J. Knap and M. Ortiz, "Nanovoid deformation in aluminum under simple shear," Acta Materialia, vol. 2005, pp. 2893 - 2900, 2005.

[46] A. Seeger, "CXXXII. The generation of lattice defects by moving dislocations, and its application to the temperature dependence of the flow-stress of F.C.C. crystals," The London, Edinburgh, and Dublin Philosophical Magazine and Journal of Science, vol. 46:382, pp. 1194 - 1217, 1955.

[47] M. Niewczas, "Intermittent plastic flow of single crystals: central problems in plasticity: a review," Materials Science and Technology, vol. 30, no. 7, pp. 739 - 757, 2014.

[48] F. Seitz, "On the generation of vacancies by moving dislocations," Advances in Physics, vol. 1, no. 1, pp. 43-90, 1952. 
[49] T. M. Smith, M. S. Hooshmand, B. Esser, F. Otto, D. W. McComb, E. P. George, M. Ghazisaeidi and M. Mills, "Atomic-scale characterization and modeling of $60^{\circ}$ dislocations in a high-entropy alloy," Acta Materialia, vol. 110, pp. 352 - 363, 2016.

[50] X. Zhang, "A continuum model for dislocation pile-up problems," Acta Materialia, vol. 128, pp. $428-439,2017$.

[51] B. Pan, Y. Shibutani, X. Zhang and F. Shang, "Effect of dislocation pile-up on size-dependent yield strength in finite single-crystal micro-samples," Journal of Applied Physics, vol. 118, 2015.

[52] S. I. Hong and C. Laird, "Mechanisms of slip mode modification in F.C.C. solid solutions," Acta Metallurgica et Materialia, vol. 38, no. 8, pp. 1581 - 1594, 1990.

[53] R. M. Latanision and A. W. Ruff Jr, "The Temperature Dependence of Stacking Fault Energy in Fe-Cr-Ni Alloys," Metallurgical Transactions, vol. 2, no. 2, pp. 505 - 509, 1971.

[54] I. A. Yakubtsov, A. Ariapour and D. D. Perovic, "Effect of Nitrogen on Stacking Fault Energy of F.C.C Iron-Based Alloys," Acta materialia, vol. 47, no. 4, pp. 1271 - 1279, 1999.

[55] N. L. Okamoto, S. Fujimoto, Y. Kambara, M. Kawamura, Z. M. T. Chen, H. Matsunoshita, K. Tanaka, H. Inui and E. P. George, "Size effect, critical resolved shear stress, stacking fault energy, and solid solution strengthening in the CrMnFeCoNi high-entropy alloy," Scientific Reports, vol. 6, 2016.

[56] C. Niu, C. R. LaRosa, J. Miao, M. J. Mills and M. Ghazisaeidi, "Magnetically-driven phase transformation strengthening in high entropy alloys," Nature Communications, vol. 9, 2018.

[57] B. Sun, N. Vanderesse, F. Fazeli, C. Scott, J. Chen, P. Bocher, M. Jahazi and S. Yue, "Discontinuous strain-induced martensite transformation related to the Portevin-Le Chatelier effect in a medium manganese steel," Scripta Materialia, vol. 133 , pp. 9 - 13, 2017.

[58] S. Allain, O. Bouaziz, T. Lebedkina and M. Lebyodkin, "Relationship between relaxation mechanisms and strain aging in an austenitic FeMnC steel," Scripta Materialia, vol. 64, pp. 741 744, 2011.

[59] A. Eisenlohr, I. Gutierrez-Urrutia and D. Raabe, "Adiabatic temperature increase associated with deformation twinning and dislocation plasticity," Acta Materialia, vol. 60, pp. 3994 - 4004, 2012. 